\title{
Regionalisation of retinopathy of prematurity (ROP) screening improves compliance with guidelines: an audit of ROP screening in the Northern Region of England
}

\author{
N G Ziakas, D G Cottrell, D W A Milligan, P M Pennefather, M A Bamashmus, \\ M P Clarke
}

\begin{abstract}
Aims-This project was designed to determine whether a coordinated regional strategy can improve the implementation of national guidelines for screening for retinopathy of prematurity (ROP), and to identify causes for failure of compliance.

Methods-Retrospective case note audit relating to two periods, 1990-1 and 1994, involving all 17 neonatal intensive care units in the Northern Region of England. Between the two periods, a regional strategy was instituted in an endeavour to improve compliance. Babies born in or admitted to the units during the study periods who were eligible for ROP screening were included. Screening performance was assessed against a standard of $100 \%$ compliance with the guidelines. In the second audit period compliance with subsidiary standards was also measured, and reasons for failure were identified.

Results-Compliance improved from $47 \%$ (262/558) in the first audit cycle to $73 \%$ $(264 / 360)$ in the second. Subgroup analysis in this second cycle indicated better compliance $(93.3 \%)$ in higher risk babies $(\leqslant 29$ weeks' gestational age). Babies transferred between units, discharged home before screening, or who failed to qualify for screening on one of the two defined criteria, were more likely to be missed. Conclusion-A carefully implemented regional approach to screening resulted in a higher uptake for babies most at risk. Simple recommendations are made to achieve further improvement in compliance with the guidelines. The wider implications for screening in other conditions and in other areas and specialties are highlighted.

(Br f Ophthalmol 2001;85:807-810)
\end{abstract}

Sight threatening retinopathy of prematurity (ROP) is a worldwide problem with a considerable geographical variation in its incidence, ${ }^{1-3}$ affecting $3.6 \%$ of babies born before 32 weeks' gestation in the Northern Region of the National Health Service in England (population approximately three million). ${ }^{4}$ The multicentre trial of cryotherapy treatment (CRYOROP) defined a threshold level of ROP at which treatment was to be administered; it resulted in a reduction of approximately 50\% in the occurrence of posterior retinal traction, folds, and/or detachments. ${ }^{5}$ Subsequent studies have shown diode laser treatment of ROP to be at least as effective as cryotherapy with fewer side effects. ${ }^{6}$

The goal of screening is to prevent unfavourable visual outcomes from ROP by detecting the more severe stages early enough to allow appropriate intervention. Protocols have been produced by national and international bodies to ensure that those babies at risk of blindness from ROP and reaching "threshold disease" are screened in time for treatment to be offered. "Threshold disease" is defined as stage 3 ROP involving 5 or more contiguous, or 8 or more cumulative, clock hours in zone 1 or 2 in the presence of congestion of the posterior pole vessels- "plus" disease. In the UK, national guidelines were produced in 1990 and revised in $1995 .^{\circ}$ They indicate that all babies born at $\leqslant 31$ weeks' gestation and/or $\leqslant 1500 \mathrm{~g}$ birth weight should be screened from 6-7 weeks of postnatal age until there is vascularisation into zone 3. While there is currently some national and international debate about the need to screen all babies meeting these criteria, ${ }^{9}$ guidelines can only be effective if compliance with their recommendations is good.

The only study in the literature analysing compliance with guidelines for ROP screening comes from a single neonatal unit in North America. ${ }^{10}{ }^{11}$ The present study describes two separate audits of compliance with the national ROP screening guidelines ${ }^{8}$ for the Northern Region of England. Defects identified in the first audit cycle were addressed by a regional committee which met in 1993 and devised and implemented a regional strategy. In the second cycle the efficacy of this strategy was assessed and recommendations made for further improvement.

\section{Materials and methods}

Each audit cycle (cycle 1 and cycle 2) involved a retrospective review and analysis of case notes of babies eligible for ROP screening born in the Northern Region of England during the study periods. In each cycle the notes from all 17 neonatal units in the region were reviewed. The first period was 1990-1 (two full years) and the second period was 1994 (one full year).

Entry criteria were slightly different in the two cycles. The first audit formed part of a 


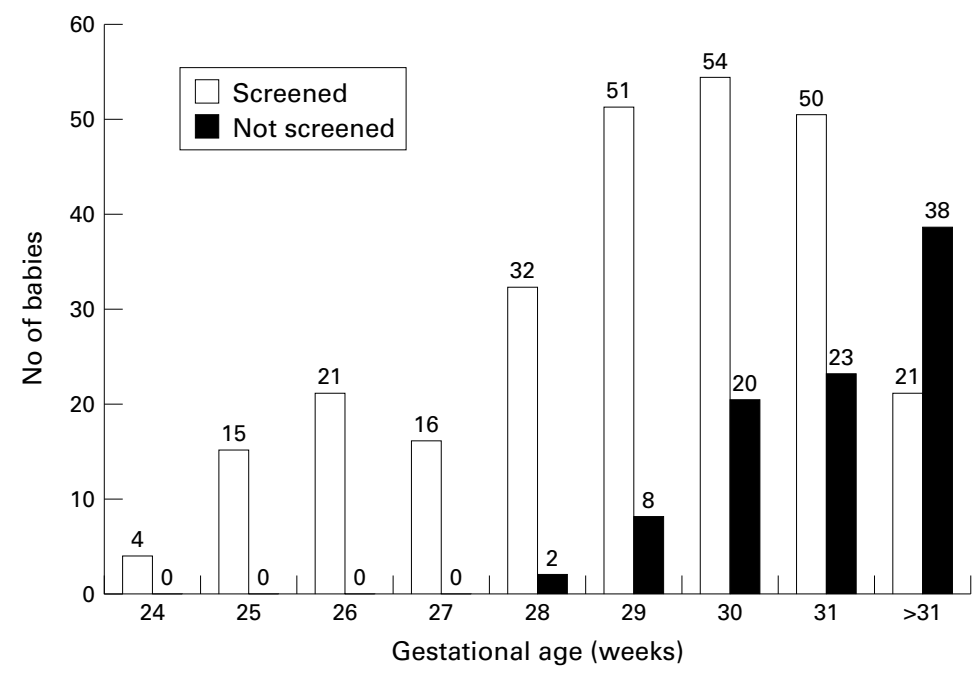

Figure 1 Number of premature babies screened in the different gestational age (GA) groups. ophthalmic specialists in the region were identified as being available to provide advice on screening to colleagues, and to offer a prompt treatment service for babies identified as having threshold disease. The importance of communication of screening requirements on discharge or transfer to another hospital was emphasised.

The case notes were scrutinised for evidence of adherence to these standards. In cycle 2, additional data on factors associated with compliance or non-compliance with the standards were sought. "High risk" babies were defined as those born at 29 weeks' gestation or less. ${ }^{413}$

\section{Results}

In all, 565 babies were eligible for inclusion in cycle 1 ; case notes were available for 558 babies $(98.8 \%)$. A total of 367 babies were eligible and 360 case notes were available $(98.1 \%)$, in cycle 2 .

separate study of global outcomes of premature birth at age 2 years, ${ }^{4}$ involving all babies who were born before 32 weeks and survived to age 2 years. In the second cycle babies with a birth weight $\leqslant 1500 \mathrm{~g}$ were also included (in order to follow the Royal College of Ophthalmologists' guidelines exactly $)^{8}$ and the audit was performed on those who survived to screening age.

AUDIT STANDARDS

Standard $A$ (cycles 1 and 2)

All babies satisfying the criteria in the guidelines should be screened.

Standard B (cycle 2 only)

Screening should start at 6-7 weeks after birth, and continue at least 2 weekly until vascularisation has progressed into zone $3 .^{812}$

Appropriate screening arrangements must be made for babies due to be transferred to another hospital or discharged home before the screening process is complete; this should include the receiving hospital being informed of the requirement for screening by the neonatal staff of the transferring hospital or, if the infant is discharged home, an appropriate outpatient appointment must be made.

After the first cycle a regional strategy for screening was circulated to all 17 neonatal units, to ophthalmologists, to paediatricians in the region, and to those responsible for regional health policy. The strategy document reiterated the guidelines, indicated that the attending paediatrician was responsible for identifying babies requiring screening, and specified a named ophthalmologist to provide screening for each neonatal unit. Additional arrangements were included in case the named ophthalmologist should be unavailable. Three

Table 1 Screening compliance for babies fulfilling only one of the guidelines' inchusion criteria compared with those that fill both

\begin{tabular}{|c|c|c|c|c|}
\hline & $>31$ weeks $/ \leqslant 1500 \mathrm{~g}$ & $\leqslant 31$ weeks $/>1500 \mathrm{~g}$ & $\leqslant 31$ weeks $/ \leqslant 1500 \mathrm{~g}$ & All babies \\
\hline Screened & $21(36 \%)$ & $55(71 \%)$ & $188(84 \%)$ & $264(73 \%)$ \\
\hline Not screened & $38(64 \%)$ & $23(29 \%)$ & $35(16 \%)$ & $96(27 \%)$ \\
\hline
\end{tabular}

Basic screening compliance

Compliance improved from $47 \%$ (262 out of 558 babies) in the first cycle to $73 \%$ (264 out of 360 babies) in the second cycle.

\section{Compliance figures in cycle 2}

Compliance rates in cycle 2 were analysed for groups subdivided into ranges of gestational age at birth (GA) and birth weight (BW). Figscreened in the different GA groups; 93\% (140 of 150) of "high risk" babies (GA $\leqslant 29$ weeks) were screened. Screening failures occurred more frequently in babies who did not fulfil one of the two entry criteria (Table 1).

\section{AUDIT STANDARD B}

Of the 264 screened babies the guidelines were followed rigidly in only $183(70 \%)$. In 75 (28\%) the screening did not accurately follow the guidelines, being started late in 51, finished early in 11 , and otherwise inadequate in 13 .

A total of 72 out of the $264(27 \%)$ babies screened developed ROP; 38 reached stage 1 ROP, 15 reached stage 2 ROP, and 19 reached stage 3 ROP. $^{13}$ Of those with stage 3,15 reached threshold disease and were treated by cryotherapy to the avascular retina.

A total of 196 babies were transferred to a different neonatal unit before completion of screening. Screening was mentioned in the transfer letter in only $87(44 \%)$ and data were unavailable in $12(6 \%)$. Screening was completed in $83(95 \%)$ of those transferred with such a letter. In those transferred without a recommendation letter for screening only 73 (75\%) were screened for ROP. Ten of the transferred babies $(5.1 \%)$ developed stage 3 ROP, a virtually identical percentage to that of

\section{AUDIT STANDARD A} ure 1 shows the number of premature babies 
non-transferred babies (5.5\%). However, five of the 14 babies (36\%) who were transferred three or more times developed threshold stage 3 disease; all of these were under 27 weeks' gestation and under $850 \mathrm{~g}$ at birth. Three of these 14 babies (21\%) were never screened, but all three were born at 30 or more weeks' gestation and were over $1300 \mathrm{~g}$.

In all, 71 babies were discharged home before 36 weeks of postmenstrual age. In 57 cases $(80.3 \%)$ the discharge summary gave no indication of arrangements for screening and none was screened after discharge. In the remaining 14 cases, an appointment was made before discharge or there was a request for screening in the discharge summary. Of these, nine babies were screened and the others either failed to attend to the clinic (three) or no follow up appointment was sent to them (two).

\section{Discussion}

Treatment of acute ROP reduces the incidence of retinal scarring and detachment with a consequent reduction in subsequent visual handicap. ${ }^{5}$ Surgical treatment of retinal detachment due to ROP, while achieving some anatomical success, results in minimal if any visual improvement. ${ }^{14}$ It is important therefore that premature infants with threshold disease are identified and treated before irretrievable damage occurs. The interval between threshold being reached and detachment occurring may only be a matter of days so for the screening programme to succeed it must be effectively implemented. Only one study in the literature has addressed this. ${ }^{1011}$

The low compliance rate in cycle 1 indicated that arrangements for screening were haphazard and unreliable. Our study therefore addressed the identification of premature infants at risk of acute ROP and the potential benefits of a coordinated regional strategy. A retrospective design was chosen to avoid the possibility that a prospective study would alert personnel to the requirement to screen for ROP, potentially only for the duration of the study, and therefore would not allow accurate assessment of the effects of the strategy. ${ }^{11}$

There are systematic differences between cycle 1 and cycle 2: cycle 1 did not include babies who were $>31$ weeks but $\leqslant 1500 \mathrm{~g}$ and excluded those babies who died before 2 years of age; cycle 2 included the birthweight criterion and all babies who survived long enough to be screened. Reanalysis of cycle 2 without the birthweight criterion increases the screening rate from $73 \%$ to $80 \%$ and magnifies the difference between the two cycles. Data from regional records indicate that 31 additional babies would have been included in cycle 1 had they not died between screening age and 2 years, and these babies might have been at higher risk of ROP and more likely to have been screened. However, even if compliance with screening were $100 \%$ in these cases, the overall compliance for cycle 1 would increase only marginally (to $50 \%$ ), which would not make a significant difference to the result.
This study indicates that there was a large improvement (from $47 \%$ to $73 \%$ ) in the ROP screening rate throughout the region following implementation of a regional strategy. Screening failures in cycle 2 were largely confined to low risk $>29$ week babies and those who failed to meet one of the screening criteria; no significant sequelae of acute ROP had subsequently been recorded in any of these cases when the case note review was performed (1996-7). The results indicate, however, that babies transferred to other hospitals are at particular risk of being missed from screening unless clear recommendations are made to the receiving unit and a mechanism exists to put these into effect. The high percentage $(36 \%)$ of babies undergoing multiple transfer who developed threshold stage 3 ROP is interesting; fortunately they were all of very early gestation and therefore unlikely to be missed from screening.

It cannot be proved that the strategy was the sole cause of the improvement as general awareness of ROP has been increasing over this time but we believe that the availability of a named ophthalmologist to paediatricians in every neonatal unit has been central to its success. It was also helpful that the strategy identified the professional responsible for initiating the screening process in each individual case (the paediatrician).

Ophthalmological review did not continue in some instances for as long as the guidelines recommend. It is not clear from this study whether this was due to rapid retinal maturation (positive identification of the lack of clinical need for further examinations) or to administrative or other problems. Screening is sometimes postponed because the baby is judged to be too sick to tolerate the stress although it was not possible positively to identify this as a reason from a retrospective notes review. This could have important medicolegal implications.

Further recommendations have been made within the region since the results of cycle 2 became available:

(1) Neonatal units are encouraged to keep a diary for prospective entry of dates for ROP screening to begin.

(2) Standard templates for discharge/transfer letters should contain a default section on ROP screening.

(3) A standard screening record form, the design of which has been agreed throughout the region, should always be used.

(4) When screening is incomplete at the time of transfer, copies of the screening records to date should be faxed to the ophthalmologist responsible for the receiving unit.

(5) Where postponement or early termination of screening occurs this should be positively identified in the case notes.

A third audit is planned in due course.

The data here relate to a single health region in the UK but the measures taken or recommended are simple in themselves and could readily be adopted and applied in other areas in order to improve compliance. 
Presented at World ROP Meeting, Taormina, Sicily, Italy in September 1997 and at the Royal College of Ophthalmologists May 1998.

Thanks to the Northern Neonatal Network team who helped with notes retrieval and to the Northern Regional Purchaser Clinical Audit Group who funded the project. The authors are also grateful to their many colleagues who allowed their patients' records to be studied.

$1 \mathrm{Ng}$ YK, Fielder AR, Shaw DE, et al. Epidemiology of retinopathy of prematurity. Lancet 1988;ii:1235-8.

Holmstrom G, El Azazi M, Jacobson L, et al. A population based, prospective study of the development of ROP in prematurely born children in the Stockholm area of Sweden. Br F Ophthalmol 1993;77:417-23.

Darlow BA. Incidence of retinopathy of prematurity in New Zealand. Arch Dis Child 1988;63:1083-6.

4 Pennefather PM, Clarke MP, Strong NP, et al. Ocular outcome in children born before 32 weeks gestation. Eye 1995;9(Suppl):26-30.

5 Cryotherapy for Retinopathy of Prematurity Cooperative Group. Multicentre trial of cryotherapy for retinopathy of Group. Multicente trith of premation 114:417-24.

6 Paysse EA, Lindsey JL, Coats DK, et al. Therapeutic outcomes of cryotherapy versus transpupillary diode laser photocoagulation for threshold retinopathy of prematurity. f AAPOS: Am Ass Pediatric Ophthalmol Strabismus 1999;3 234-40.
7 Screening examination of premature infants for Retinopathy of Prematurity. A joint statement of the American Academy of Pediatrics, the American Association for Pediatric Ophthalmology and Strabismus, and the American Academy of Ophthalmology. Ophthalmology 1997;104:888-9.

8 Wilkinson AR, Clark D, Fielder A, et al. Retinopathy of prematurity: guidelines for screening and treatment. The report of a joint working party of the Royal College of Ophthalmologists and the British Association of Perinatal Medicine. Early Human Development 1996;46:239-58.

9 Schalij-Delfos NE, Zijlmans BL, Wittebol-Post D, et al. Screening for retinopathy of prematurity: do former guide-
lines still apply? $\mathcal{F}$ Pediatr Ophthalmol Strabismus 1996;33: 35-8.

10 Trainor S, White GL Jr, Kivlin JD. Compliance with a standard of care for retinopathy of prematurity in one neonatal intensive care unit. $\mathcal{F}$ Pediatr Ophthalmol Strabismus 1988;25:237-9.

11 Trainor S, White GL, Kivlin JD, et al. Follow up retrospective study of compliance with a standard of care for retinopathy of prematurity in one neonatal intensive care unit. $\mathcal{F}$ Pediatr Ophthalmol Strabismus 1989;285-7.

12 The Committee for the Classification of Retinopathy of Prematurity. An international classification of retinopathy of prematurity. Arch Ophthalmol 1984;102:1130-4.

13 Goble RR, Jones HS, Fielder AR Are we screening too many babies for retinopathy of prematurity? Eye 1997;11:509-14.

14 Quinn GE, Dobson V, Barr CC, et al. Visual acuity in infants after vitrectomy for severe retinopathy of prematurity. $O p h$ thalmology 1991;98:5-13.

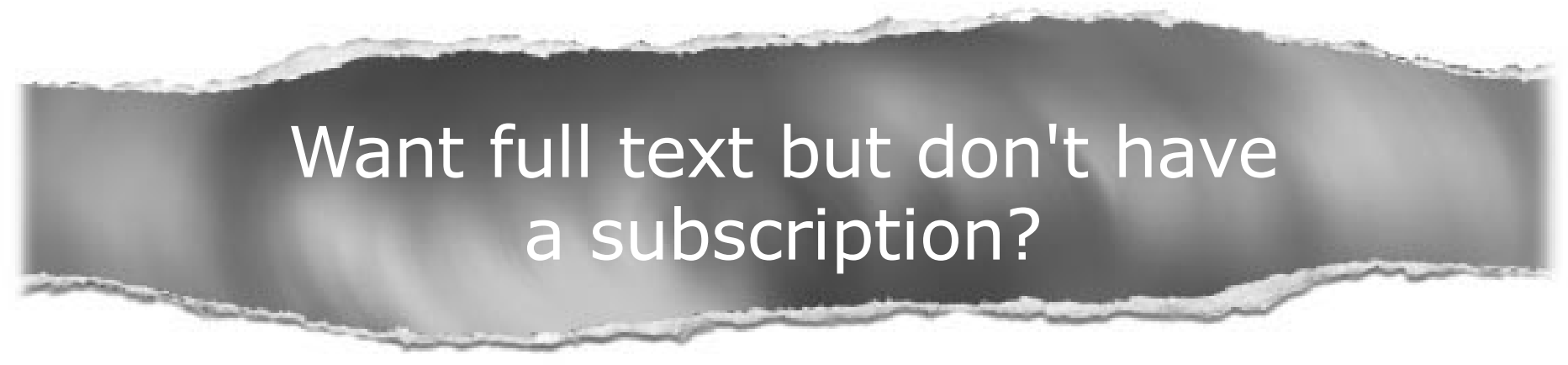

\section{Pay per view}

For just \$8 you can purchase the full text of individual articles using our secure online ordering service. You will have access to the full text of the relevant article for 48 hours during which time you may download and print the pdf file for personal use.

www.bjophthalmol.com 